\title{
Capacidade de predição da disponibilidade de fósforo em solo com aplicação de fosfato solúvel e natural
}

\section{Capacity to predict the availability of phosphorus in soils with soluble and natural phosphate}

\author{
Clovisson Menotti Boeira de OLIVEIRA ${ }^{1 ; 2}$; Luciano Colpo GATIBONI ${ }^{3}$; Paulo Roberto ERNANI ${ }^{4}$; \\ Gustavo BOITT' ${ }^{5}$; Gustavo BRUNETTO 6
}

${ }^{1}$ Parte da dissertação de mestrado do primeiro autor. Trabalho realizado com recursos da Capes e do CNPq

2 Engenheiro Agrônomo, Doutorando em Manejo do Solo. Centro de Ciências Agroveterinárias, Universidade do Estado de Santa Catarina, Avenida Luiz de camões, 2090, Conta Dinheiro, 88520-000, Lages, Santa Catarina, Brasil. clovissonboeira@yahoo.com.br;

${ }^{3}$ Engenheiro Agrônomo, Doutor em Agronomia, Professor do Centro de Ciências Agroveterinárias, Bolsista em produtividade do CNPq, Universidade do Estado de Santa Catarina, Avenida Luiz de camões, 2090, Conta Dinheiro, 88520-000, Lages, Santa Catarina, Brasil. gatiboni@cav.udesc.br;

${ }^{4}$ Engenheiro Agrônomo, PhD em Fertilidade do Solo e Nutrição de Plantas. Professor do Centro de Ciências Agroveterinárias, Bolsista em produtividade do CNPq, Universidade do Estado de Santa Catarina, Avenida Luiz de camões, 2090, Conta Dinheiro, 88520-000, Lages, Santa Catarina, Brasil. a2pre@cav.udesc.br;

${ }^{5}$ Engenheiro Agrônomo, Mestrando em Ciência do Solo, Centro de Ciências Agroveterinárias, Universidade do Estado de Santa Catarina, Avenida Luiz de camões, 2090, Conta Dinheiro, 88520-000, Lages, Santa Catarina, Brasil. a2gbo@cav.udesc.br;

${ }^{6}$ Autor para correspondência; Eng. Agrônomo; Doutor em Ciência do Solo; Professor Adjunto no Departamento de Solos; Centro de Ciências Rurais; Universidade Federal de Santa Maria (UFSM). Av. Roraima, 1000, Camobi, CEP: 97105-340, Santa Maria - RS, Brasil. Telefone: (55) 3220-8108. brunetto.gustavo@gmail.com

Recebido em: 24-07-2014; Aceito em: 21-06-2015

\section{Resumo}

Em solos adubados com fosfatos naturais, não é recomendado o uso do método Mehlich 1 para a avaliação da disponibilidade de fósforo $(\mathrm{P})$, por causa da superestimação de seus teores disponíveis às plantas (CQFSRS/SC, 2004). O objetivo do trabalho foi avaliar a capacidade de predição da disponibilidade de P pelos métodos Mehlich 1, Mehlich 3 e resina trocadora de ânions em lâminas (RTAl) e em esferas (RTAe), em solo adubado com fosfato solúvel e natural. O experimento foi realizado em casa de vegetação, usando um Cambissolo Húmico. Os tratamentos foram arranjados em esquema fatorial $2 \times 3 \times 4$, em que o primeiro fator era representado pelos pHs do solo $(4,4 \mathrm{e} \mathrm{pH} \mathrm{6,5),} \mathrm{o} \mathrm{segundo} \mathrm{por} \mathrm{fontes} \mathrm{de} \mathrm{fósforo} \mathrm{(testemunha,} \mathrm{superfosfato} \mathrm{triplo} \mathrm{e} \mathrm{fosfato}$ natural de Arad) na quantidade equivalente a $100 \mathrm{~kg}^{-1}$ de $\mathrm{P}_{2} \mathrm{O}_{5}$, e o terceiro pelos métodos de extração (Mehlich 1, Mehlich 3, RTAl e RTAe). Cultivou-se milheto por 43 dias para posterior determinação da massa da matéria seca produzida, P absorvido e o teor de P disponível. O Mehlich 3, RTAl e RTAe são métodos eficientes na determinação de $\mathrm{P}$ no solo, na ocasião da aplicação de fosfato natural de Arad, enquanto o Mehlich 1 é inadequado para a determinação da disponibilidade de $\mathrm{P}$, na ocasião da aplicação de fosfato natural de Arad.

Palavras-chave adicionais: fosfato de rocha; métodos de análise; solubilidade; superestimação.

\begin{abstract}
The amount of available phosphorus $(\mathrm{P})$ in soils fertilized with rock phosphates is overestimated when the Mehlich 1 method is used to extract $P$ (CQFS-RS/SC, 2004). The objective of this work was to evaluate the capacity of Mehlich 1, Mehlich 3 and Anion Exchange Resin to predict available $P$ to millet plants in a soil fertilized with soluble phosphate or rock phosphate. The experiment was carried out in a greenhouse using a Humic Cambisol. Treatments included a factorial combination of two soil $\mathrm{pH}$ levels (4.4 and 6.5), two $\mathrm{P}$ sources (triple superphosphate and Arad rock phosphate and the control, without $\mathrm{P}$ ), in the amount of $\mathrm{P}$ added equivalent to $100 \mathrm{~kg} \mathrm{ha}^{-1} \mathrm{P}_{2} \mathrm{O}_{5}$, and four extraction methods (Mehlich 1, Mehlich 3, RTAl and RTAe). The four replications were arranged in a completely randomized experimental design and millet plants was grow during 43 days. Anion exchange resin, in strips or in spheres, and Mehlich 3 are efficient methods for the determination of $P$ in soil fertilized with Arad rock phosphate, while Mehlich 1 is inappropriate.
\end{abstract}

Additional keywords: methods of soil analysis; overestimation; rock phosphate; solubility. 


\section{Introdução}

A condição eficaz para aumentar o fósforo $(P)$ disponível às plantas é a aplicação de adubos fosfatados, que constitui a principal fonte do nutriente para as culturas anuais. A rocha fosfática, após concentração, porém com lenta solubilização, é denominada como fosfato natural. Mesmo com menor eficiência agronômica, essas fontes naturais têm sido usadas pela busca por fontes alternativas de menor custo econômico, e isso tem aumentado o consumo de fontes de fósforo de baixa solubilidade, como os fosfatos naturais reativos. Algumas condições edáficas favoráveis à solubilização dos fosfatos naturais são o $\mathrm{pH}$ baixo e a presença simultânea de dreno de $\mathrm{P}$ e dreno de cálcio $(\mathrm{Ca})$, que são eficientes fatores do solo que aceleram o processo de dissolução de fosfato natural (Robinson \& Syers, 1990).

A utilização de diferentes fontes de $P$ nos solos necessita que sejam utilizados extratores versáteis que estimem com precisão o $\mathrm{P}$ disponível do solo, ou que seja capaz de se correlacionar com a absorção da planta. O método Mehlich 1, que age no solo por dissolução ácida, consiste na solubilização em ordem decrescente de efetividade das formas de $P$ ligadas a cálcio $(\mathrm{Ca})$, alumínio $(\mathrm{Al})$ e ferro $(\mathrm{Fe})$, e, desta forma, como desvantagem, pela extração preferencial de compostos de $\mathrm{Ca}$, superestima os teores disponíveis em solos com presença de fosfatos de Ca como mineral primário (Mehlich, 1978), e também nos que receberam adubação com fosfatos naturais (Gatiboni et al., 2003; Bortolon et al., 2011).

Devido aos problemas de superestimação de $\mathrm{P}$ em solos calcários ou com adição de fosfatos naturais, Mehlich (1984) propôs modificações do método criando o método Mehlich 3, tendo em conta como vantagem a extração simultânea de outros macronutrientes e micronutrientes. Para evitar os problemas de superestimação da disponibilidade de $P$, podem-se ainda utilizar métodos sensíveis apenas às formas de $\mathrm{P}$ acessíveis às plantas. Proposto por Amer et al. (1955), a Resina Trocadora de Ânions (RTA) em lâminas ou esferas possui sítios permanentes de adsorção de ânions. Uma característica favorável ao método é que pode ser usado também em solo alcalino, não superestimando a disponibilidade do $\mathrm{P}$ em solos que receberam fosfato natural (Silva \& Raij, 1999).

O problema estabelecido neste trabalho é que há a superestimação do $\mathrm{P}$ disponível pelo método Mehlich 1 em solos com a adição de fosfatos naturais, enquanto os métodos Mehlich 3 e RTA, em razão de sua composição química e modo de ação, podem apresentar melhor precisão em solos que receberam a adubação com diferentes fontes de fosfatos (Freitas et al., 2013). O objetivo com este trabalho foi avaliar a capacidade de predição da disponibilidade do $P$ pelos métodos Mehlich 1, Mehlich 3 e RTA em lâminas e em esferas, em solo com dois níveis de $\mathrm{pH}$ e que receberam a adição de fosfato solúvel e fosfato natural.

\section{Material e métodos}

O experimento foi conduzido no Centro de Ciências Agroveterinárias da Universidade do Estado de Santa Catarina (CAV/UDESC), em Lages (SC). O solo utilizado foi um Cambissolo Húmico Alumínico léptico, coletado na camada de $0-20 \mathrm{~cm}$ de profundidade, em área de campo nativo. Após secagem em estufa com circulação forçada de ar a $65{ }^{\circ} \mathrm{C}$ por $48 \mathrm{~h}$ e passado em peneira com malha de $2 \mathrm{~mm}$, foi realizada a análise física do solo, que apresentou $250 \mathrm{~g} \mathrm{~kg}^{-1}$ de argila; $460 \mathrm{~g} \mathrm{~kg}^{-1}$ de silte e $290 \mathrm{~g} \mathrm{~kg}^{-1}$ de areia, determinado pelo método da pipeta (Embrapa, 1997), e a análise química do solo, conforme metodologia proposta por Tedesco et al. (1995), que apresentou pH em água de 4,4; Índice SMP de 4,7; 14,8 mg kg-1 de P disponível; $121 \mathrm{mg} \mathrm{kg}^{-1}$ de $\mathrm{K}$ trocável; $3,2 \mathrm{cmol}_{\mathrm{c}} \mathrm{kg}^{-1}$ de $\mathrm{Ca}$ trocável; $1,4 \mathrm{cmol}_{\mathrm{c} \mathrm{kg}}{ }^{-1}$ de $\mathrm{Mg}$ trocável; $4,8 \mathrm{cmol}_{\mathrm{c} \mathrm{kg}}{ }^{-1}$ de $\mathrm{Al}$ trocável e $48 \mathrm{~g} \mathrm{~kg}^{-1}$ de matéria orgânica.

Os tratamentos constaram de dois níveis de $\mathrm{pHs}$ do solo, duas fontes de $\mathrm{P}$ e quatro métodos de extração, mais testemunha. Os pHs utilizados foram 4,4 (sem correção da acidez) e correção do pH até 6,5, pela adição de calcário Filler na dose recomendada pelo índice de SMP (CQFS-RS/SC, 2004). O solo foi incubado em laboratório por 30 dias, em baldes plásticos, com umidade próxima a $80 \%$ da capacidade de campo e revolvimento a cada três dias. Após a incubação, o solo foi novamente seco em estufa com circulação forçada de ar a $65^{\circ} \mathrm{C}$, por $48 \mathrm{~h}$, moído e passado em peneira com malha de $2 \mathrm{~mm}$. As fontes de $\mathrm{P}$ utilizadas foram superfosfato triplo (SFT), com $42 \%$ de $\mathrm{P}_{2} \mathrm{O}_{5}$ total e fosfato natural de Arad (FN), com $33 \% \mathrm{P}_{2} \mathrm{O}_{5}$ total, as quais foram previamente moídas com granulometria final entre 0,3 e $0,5 \mathrm{~mm}$, mais testemunha sem $P$. Para a aplicação das fontes de fosfatos, considerouse o volume da camada arável em um hectare, equivalente a 2.000 .000 de quilogramas, com $20 \mathrm{~cm}$ de profundidade. A dose de $\mathrm{P}$ utilizada foi de $100 \mathrm{~kg} \mathrm{ha}^{-1}$ de $\mathrm{P}_{2} \mathrm{O}_{5}$, equivalente a $0,273 \mathrm{~g} \mathrm{~kg}^{-1}$ solo de SFT e $0,347 \mathrm{~g} \mathrm{~kg}^{-1}$ solo de FN, com aplicação incorporada ao solo, para o cultivo de milheto. Os métodos de extração foram Mehlich 1, Mehlich 3, RTA em lâminas e RTA em esferas. O experimento foi arranjado em esquema fatorial $(2 \times 3 \times 4)$ no delineamento inteiramente casualizado, com quatro repetições, e conduzido em casa de vegetação.

Para o cultivo, as amostras foram acondicionadas em vasos com capacidade de $1,0 \mathrm{~kg}$ e foram semeadas cinco sementes pré-germinadas de milheto (Penninsetum americanum) por vaso. A pré-germinação foi realizada em laboratório, em folha de papel-filtro umedecido, mantendo em temperatura ambiente. A semeadura de sementes pré-germinadas foi para uniformizar o crescimento inicial das plantas, para estabelecer um período de tempo semelhante entre as plantas nos vasos. Aos 8 dias após a implantação, fez-se um desbaste, deixando-se duas 
plantas uniformes por vaso e efetuou-se a adubação com 20 e $25 \mathrm{mg} \mathrm{kg}^{-1}$ solo de $\mathrm{N}$ e K por vaso, equivalendo a $89 \mathrm{~kg} \mathrm{ha}^{-1}$ de ureia a $100 \mathrm{~kg} \mathrm{ha}^{-1}$ de cloreto de potássio, respectivamente, conforme recomendação (CQFS-RS/SC, 2004). A umidade do solo foi mantida próxima a $80 \%$ da capacidade de campo, por meio de regas diárias com água destilada para repor a água perdida por evapotranspiração, fazendo-se o monitoramento por meio de pesagens diárias dos vasos.

As plantas foram cultivadas apenas durante 0 estádio vegetativo e, aos 43 dias após a semeadura foi realizada a coleta da parte aérea e das raízes. Após a coleta das raízes, elas foram separadas do solo e lavadas com água destilada. O material vegetal (parte aérea e raízes) foi seco em estufa com circulação forçada de ar a $65^{\circ} \mathrm{C}$, por $48 \mathrm{~h}$, e determinada a produção de massa da matéria seca (MMS). As amostras da parte aérea e de raízes foram moídas, submetidas à digestão sulfúrica e determinados os teores de fósforo no tecido vegetal, conforme Tedesco et al. (1995). Tomando por base a produção total de MMS, foi calculada a eficiência agronômica (EA) do fosfato natural de Arad pela seguinte equação: $E A=(M M S$ com fosfato natural de Arad - MMS na testemunha / MMS com superfosfato triplo - MMS na testemunha) $\times 100$.

Após a coleta das plantas, as amostras de solo acondicionados nos vasos foram retiradas da casa de vegetação e colocadas para secar em estufa com circulação forçada de ar a $65 \stackrel{\circ}{\circ}$, por $48 \mathrm{~h}$, moídas e tamisadas em peneira com malha de $2 \mathrm{~mm}$, e submetidas à extração do fósforo pelos métodos de Mehlich 1, Mehlich 3, RTA, em esferas saturadas com $\mathrm{NaHCO}_{3}$, e a RTA em lâminas, descritos respectivamente por Mehlich (1953), Mehlich (1984), Raij \& Quaggio (1983) e Tedesco et al. (1995). O fósforo dos extratos foi determinado segundo a metodologia de Murphy \& Riley (1962).

Para a análise estatística, foi adotado um modelo linear de análise da variância considerando os dados obtidos a partir de um delineamento em parcelas subdivididas. As parcelas constituíram-se nos diferentes valores de pHs; e as subparcelas, nas diferentes fontes de fósforo empregadas. As estimativas dos parâmetros do modelo foram baseadas na teoria geral de modelos lineares de Littel et al. $(1991,2006)$ e testadas através dos testes $\mathrm{F}$ e t, a $5 \%$ de probabilidade. As comparações entre os valores médios das variáveis analisadas para os diferentes valores de $\mathrm{pH}$, em cada fonte de fósforo, e para as fontes em cada valor de $\mathrm{pH}$ utilizado, foram efetuadas com o uso do teste de Bonferroni (Steel et al., 1997). As análises foram conduzidas usando-se os procedimentos GLM e MIXED do software SAS ${ }^{\circledR}$ (2003). Também foi realizado teste de correlação simples de Pearson entre fósforo extraído pelos métodos e produção de massa da matéria seca e $\mathrm{P}$ absorvido pelas plantas.

\section{Resultados e discussão}

A ineficiência do método Mehlich 1 em estimar a disponibilidade de $\mathrm{P}$ em solos que receberam fosfatos naturais é bem conhecida, tanto que, no manual de adubação e de calagem para os solos do Sul do Brasil (CQFS-RS/SC, 2004), recomenda-se a substituição da análise de $\mathrm{P}$ por Mehlich 1 pela resina, nos solos que foram adubados com fosfatos de baixa solubilidade.

Nos tratamentos adubados com superfosfato triplo, o método Mehlich 1 extraiu mais $P$ que os demais, com exceção ao Mehlich 3 no solo corrigido até $\mathrm{pH} 6,5$, onde não foram diferentes (Tabela 1).

Quando comparados os teores extraídos por cada método nos diferentes tratamentos de adubação fosfatada, pode ser observado (Tabela 1) que, para os métodos Mehlich 3, RTAl e RTAe, em ambos os pHs, os maiores teores são encontrados no tratamento SFT, seguido dos tratamentos Arad e testemunha, que não diferem entre si. Esses resultados são coerentes com a produção de MMS e P absorvido (Tabela 2). Por outro lado, para o método Mehlich 1, os teores no tratamento com fosfato natural são maiores que para o SFT, e este, maior que o da testemunha, em ambos os pHs estudados, estando este comportamento incoerente com os resultados observados na Tabela 2.

$\mathrm{Na}$ Tabela 1, onde são apresentados os teores de $\mathrm{P}$ extraídos pelos métodos avaliados, pode ser observado que os quatros métodos extraíram quantidades semelhantes de $P$ no solo sem a aplicação do nutriente, tanto em $\mathrm{pH} 4,4$ quanto em $\mathrm{pH}$ 6,5 . Para Schlindwein et al. $(2011,2013)$, os métodos de Mehlich 1, Mehlich 3 e RTA sem aplicação de P apresentaram comportamento semelhante na extração de $P$. Nos tratamentos que receberam fosfato natural de Arad, o método Mehlich 1 extraiu mais $P$ que os outros (Mehlich 3 e RTAl e RTAe), os quais não diferiram entre si, para ambos os $\mathrm{pHs}$. $\mathrm{A}$ quantidade de $\mathrm{P}$ extraído por Mehlich 1 foi da ordem de 36 a $38 \mathrm{mg} \mathrm{kg}^{-1}$, enquanto para os outros métodos, os valores situaram-se entre 5 e $14 \mathrm{mg} \mathrm{kg}^{-1}$. Santos \& Kliemann (2005), trabalhando com solos com variação nos conteúdos de argila $\left(120 \mathrm{~g} \mathrm{~kg}^{-1}\right.$ a $580 \mathrm{~g} \mathrm{~kg}^{-1}$ ), verificaram que Mehlich 1 extraiu quantidades bem superiores de $P$ que os extratores Mehlich 3 e resina. Essa maior extração deve-se ao caráter ácido do extrator (Santos \& Kliemann, 2005), cujos ácidos fortes de sua composição $\left(\mathrm{HCl}\right.$ e $\left.\mathrm{H}_{2} \mathrm{SO}_{4}\right)$ provocam a dissolução da apatita que compõe a rocha fosfática (Novais \& Smyth, 1999; Schlindwein et al., 2011; Souza, 2013). É importante ressaltar que isso se trata de um erro analítico do método, já que parte do $\mathrm{P}$ contabilizado como disponível, na verdade, não está acessível às plantas (Silva \& Raij, 1996; Rossi et al., 1999), o que pode ser comprovado pela baixa produção de MMS e $\mathrm{P}$ absorvido observada nos tratamentos com uso de fosfato natural (Tabela 2). 
Pelo apresentado na Tabela 1, as modificações introduzidas no método Mehlich 3 , com a presença do íon fluoreto complexando os sítios de ligação do P nas formas P-Al e P-Fe e a acidez da solução, mostram a efetividade da quantificação do $P$ disponível do solo, evidenciando a adequabilidade do Mehlich 3 com a aplicação de fosfato natural.

Tabela 1 - Teores de fósforo extraído no solo pelos métodos Mehlich 1, Mehlich 3, resina de troca aniônica (RTA) em lâminas e RTA em esferas, após a aplicação de fonte natural e fonte solúvel de $\mathrm{P}$ em função da variação do $\mathrm{pH}^{(1)}$. Phosphorus content estimated after extraction with Mehlich 1 solution, Mehlich 3 solution, anion exchange resin (RTA) in sheets, or RTA in beads, in soil with two pH levels and fertilized with Arad rock phosphate or triple superphosphate ${ }^{(1)}$.

\begin{tabular}{|c|c|c|c|c|c|c|}
\hline \multirow[b]{2}{*}{ Métodos de extração } & \multicolumn{3}{|c|}{$\mathrm{pH} 4,4$} & \multicolumn{3}{|c|}{$\mathrm{pH} 6,5$} \\
\hline & Testemunha & $\begin{array}{l}\text { Fosfato } \\
\text { de Arad }\end{array}$ & $\begin{array}{c}\text { Superfosfato } \\
\text { triplo }\end{array}$ & Testemunha & $\begin{array}{l}\text { Fosfato } \\
\text { de Arad }\end{array}$ & $\begin{array}{l}\text { Superfosfato } \\
\text { triplo }\end{array}$ \\
\hline \multicolumn{7}{|c|}{ - } \\
\hline Mehlich 1 & $10,66 \mathrm{aC}$ & 36,30 aA & $26,93 \mathrm{aB}$ & $7,52 \mathrm{aC}$ & 38,16 aA & $20,92 \mathrm{aB}$ \\
\hline Mehlich 3 & $9,84 \mathrm{aB}$ & $14,09 \mathrm{bB}$ & $20,40 \mathrm{bA}$ & $7,66 \mathrm{aB}$ & $9,07 \mathrm{bB}$ & $16,63 \mathrm{aA}$ \\
\hline RTA em lâminas & $6,77 \mathrm{aB}$ & $8,88 \mathrm{bAB}$ & $13,60 \mathrm{cA}$ & $5,22 \mathrm{aB}$ & $8,09 \mathrm{bB}$ & $16,26 a b A$ \\
\hline RTA em esferas & $6,19 \mathrm{aB}$ & $9,79 \mathrm{bB}$ & $16,23 \mathrm{bcA}$ & $4,38 \mathrm{aB}$ & $5,32 \mathrm{bB}$ & $10,60 \mathrm{bA}$ \\
\hline
\end{tabular}

(1) Médias seguidas de letras iguais, minúscula na coluna entre os métodos de extração para cada fonte de $P$, e maiúscula na linha entre as fontes de P para cada método de extração, não diferem entre si, pelo teste de Bonferroni: $(p<0,05)$.

A produção de massa da matéria seca (MMS) da parte aérea variou de $0,92 \mathrm{~g}$ vaso-1 no tratamento testemunha até $3,76 \mathrm{~g}$ vaso $^{-1}$ no tratamento com correção da acidez e aplicação de SFT (Tabela 2). A produção de massa da matéria seca da parte aérea foi maior com a aplicação de SFT nos dois valores de pHs avaliados, mostrando a resposta do milheto à adição de $\mathrm{P}$ em formas solúveis. Para a produção de massa da matéria seca das raízes, a aplicação de fosfato natural de Arad em solo sem correção da acidez proporcionou maior produção de massa da matéria seca das raízes $\left(1,65 \mathrm{~g}\right.$ vaso $\left.^{-1}\right)$, que nos tratamentos- testemunha $\left(0,81 \mathrm{~g} \mathrm{vaso}^{-1}\right)$ ou com correção da acidez e uso de fosfato natural de Arad $\left(0,61 \mathrm{~g} \mathrm{vaso}^{-1}\right)$.

Na soma de produção de massa da matéria seca da parte aérea e das raízes, os resultados experimentais seguiram o padrão normal discutido na literatura (Novais et al., 2007; Gatiboni et al., 2008), onde os fosfatos naturais têm desempenho intermediário entre a testemunha sem $\mathrm{P}$ e os fosfatos solúveis (neste caso, SFT). Porém, se for utilizada calagem para a correção do $\mathrm{pH}$ do solo, a dissolução do fosfato natural de Arad é diminuída, fazendo com que seu desempenho fique semelhante à testemunha e inferior ao fosfato solúvel (Gatiboni et al., 2003).

No solo sem correção da acidez, a produção de massa da matéria seca (parte aérea + raízes) aumentou de $1,73 \mathrm{~g}$ vaso-1 na testemunha para $3,67 \mathrm{e}$ $5,09 \mathrm{~g}$ vaso- ${ }^{-1}$ nos tratamentos com a aplicação de FN e $\mathrm{SFT}$, respectivamente. Já no solo com pH corrigido até 6,5 , a produção foi semelhante entre testemunha e FN $\left(1,72\right.$ e $1,67 \mathrm{~g}$ vaso $\left.^{-1}\right)$, mas inferiores ao SFT, com produção de $5,67 \mathrm{~g}$ vaso $^{-1}$.

$\mathrm{Na}$ comparação entre os pHs dentro de cada fonte de $\mathrm{P}$, só foi observado diferença para o fosfato natural de Arad (Tabela 2), cuja produção de MMS de raízes e parte aérea + raízes foi significativamente menor no solo com correção da acidez, em função da baixa eficiência deste fertilizante em solos de elevado pH (Novais \& Smyth, 1999).

A eficiência agronômica (EA) do fosfato natural de Arad foi de 57,7\% no solo com pH 4,4 (sem correção da acidez) e de $0 \%$ no solo com pH corrigido a 6,5. Essa EA do fosfato natural é devido ao curto tempo de cultivo, sendo que, em períodos com maior tempo, o efeito residual dos fosfatos naturais é evidenciado em relação à solubilidade.

O P acumulado na parte aérea variou de 0,73 a $3,73 \mathrm{mg}$ vaso $^{-1}$; e nas raízes, de 0,63 a $1,99 \mathrm{mg} \mathrm{vaso}^{-1}$ (Tabela 2). O P acumulado na parte aérea e nas raízes refletiu na produção de MMS, onde os efeitos dos tratamentos foram similares entre a produção de MMS e o $\mathrm{P}$ acumulado, evidenciando a resposta da planta à aplicação de $\mathrm{P}$. Na parte aérea, a aplicação de SFT teve o maior valor, com 3,73 e $3,08 \mathrm{mg}$ vaso $^{-1}$ no pH 4,4 e no pH 6,5, respectivamente, sendo maiores que as quantidades absorvidas nos outros tratamentos (testemunha e Arad). Com a aplicação de FN, houve diminuição significativa no $\mathrm{P}$ acumulado entre os $\mathrm{pHs}$, de 1,71 para $0,91 \mathrm{mg} \mathrm{vaso}^{-1}$, do $\mathrm{pH} 4,4$ para o $\mathrm{pH} 6,5$.

$\mathrm{O}$ acúmulo de $\mathrm{P}$ nas raízes não diferiu em $\mathrm{pH}$ 4,4 entre as fontes avaliadas, porém em pH 6,5 a aplicação de SFT favoreceu a maior produção de MMS com 1,99 g vaso-1, diferindo significativamente dos demais tratamentos (Tabela 2). Com a variação no $\mathrm{pH}$, diferiram significativamente as fontes $\mathrm{FN}$ e SFT, destacando que, para o FN, houve a diminuição de $60 \%$ no $\mathrm{P}$ acumulado nas raízes, de 1,20 para $0,48 \mathrm{mg} \mathrm{vaso}^{-1} \mathrm{com}$ o aumento do $\mathrm{pH}$. Esse valor condiz com a diminuição da MMS de raízes, que foi $63 \%$ com o aumento do $\mathrm{pH}$.

$\mathrm{O}$ acumulado de $\mathrm{P}$ na parte aérea + raízes 
apresentou o mesmo comportamento observado para a MMS. Houve diferença significativa entre as fontes em $\mathrm{pH} 4,4$, destacando a aplicação de SFT, com $5,06 \mathrm{mg} \mathrm{vaso}^{-1}$. A variação no $\mathrm{pH}$ não interferiu no acúmulo de P com a aplicação de SFT; todavia, com a aplicação de $\mathrm{FN}$, houve diminuição de $52 \%$ do $\mathrm{P}$ acumulado, com valores de 2,91 e $1,39 \mathrm{mg}^{\text {vaso- }}{ }^{-1}$ para o $\mathrm{pH} 4,4$ e pH 6,5, respectivamente.

Tabela 2 - Produção de massa da matéria seca e de fósforo absorvido por plantas de milheto cultivadas em casa de vegetação, submetidos a dois níveis de pHs do solo e aplicação de fosfato natural de Arad e superfosfato triplo(1). Shoot biomass and total phosphorus of pearl millet plants grown in greenhouse in soil with two $\mathrm{pH}$ values and fertilized with Arad rock phosphate or triple superphosphate(1).

\begin{tabular}{|c|c|c|c|c|c|}
\hline \multirow{2}{*}{$\begin{array}{l}\text { Componentes } \\
\text { da planta }\end{array}$} & \multirow{2}{*}{ Fontes de P } & \multicolumn{2}{|c|}{ Massa da matéria seca } & \multicolumn{2}{|c|}{$\mathrm{P}$ acumulado } \\
\hline & & $\mathrm{pH} \mathrm{4,4}$ & $\mathrm{pH} 6,5$ & $\mathrm{pH} \mathrm{4,4}$ & $\mathrm{pH} 6,5$ \\
\hline & & \multicolumn{2}{|c|}{-------- $\left(\right.$ g vaso-1) $^{-1}$} & \multicolumn{2}{|c|}{ - $\left(\mathrm{mg} \mathrm{vaso}^{-1}\right)$-.-. } \\
\hline & Testemunha & $0,92 \mathrm{bA}$ & $1,05 \mathrm{bA}$ & $0,97 \mathrm{bA}$ & $0,73 \mathrm{bA}$ \\
\hline \multirow[t]{2}{*}{ Parte aérea } & Fosfato natural de Arad & $2,02 \mathrm{abA}$ & $1,06 \mathrm{bA}$ & $1,71 \mathrm{bA}$ & $0,91 \mathrm{bB}$ \\
\hline & Superfosfato triplo & $3,29 a A$ & $3,76 \mathrm{aA}$ & 3,73 aA & 3,08 aA \\
\hline \multirow{3}{*}{ Raízes } & Testemunha & $0,81 \mathrm{bA}$ & $0,67 \mathrm{bA}$ & $0,64 \mathrm{aA}$ & $0,63 \mathrm{bA}$ \\
\hline & Fosfato natural de Arad & $1,65 \mathrm{aA}$ & $0,61 \mathrm{bB}$ & $1,20 \mathrm{aA}$ & $0,48 \mathrm{bB}$ \\
\hline & Superfosfato triplo & $1,80 \mathrm{aA}$ & $1,91 \mathrm{aA}$ & $1,33 \mathrm{aB}$ & 1,99 aA \\
\hline \multirow{3}{*}{ Parte aérea + raízes } & Testemunha & $1,73 \mathrm{cA}$ & $1,72 \mathrm{bA}$ & $1,61 \mathrm{cA}$ & $1,36 \mathrm{bA}$ \\
\hline & Fosfato natural de Arad & $3,67 \mathrm{bA}$ & $1,67 \mathrm{bB}$ & $2,91 \mathrm{bA}$ & $1,39 \mathrm{bB}$ \\
\hline & Superfosfato triplo & 5,09 aA & $5,67 \mathrm{aA}$ & 5,06 aA & $5,07 \mathrm{aA}$ \\
\hline
\end{tabular}

(1) Médias seguidas de letras iguais, minúsculas na coluna entre as fontes, e maiúsculas na linha entre os pHs, não diferem entre si, pelo teste de Bonferroni: $(p<0,05)$.

$\mathrm{Na}$ Tabela 3, podem ser observados os baixos índices de correlação entre os teores de $\mathrm{P}$ no solo extraído pelo método Mehlich 1 e o $\mathrm{P}$ acumulado pela planta $\left(r=0,194^{\text {ns }}\right)$, e também com a produção de massa da matéria seca $\left(r=0,180^{\text {ns }}\right)$, apontando que este extrator de fósforo é inadequado quando se utiliza o fosfato natural, pois superestima os teores extraídos de P. Rossi et al. (1999) mostraram maiores valores de $P$ extraído pelo método Mehlich 1 para os fosfatos naturais, comparativamente aos métodos Mehlich 3 e resina.

A efetividade da quantificação do $\mathrm{P}$ disponível do solo pelo Mehlich 3 é apresentada na Tabela 3 pela obtenção da melhor correlação entre a concentração de $\mathrm{P}$ no solo extraído pelo método
Mehlich 3 e a produção de massa da matéria seca das plantas de milheto $\left(r=0,914^{* *}\right)$ e para o $\mathrm{P}$ acumulado na parte aérea e nas raízes $\left(r=0,806^{* *}\right)$. Dados observados por Corrêa et al. (2008) mostraram que o método Mehlich 3 é mais adequado para determinar o $\mathrm{P}$ no solo com aplicação de fosfato natural. As correlações dos métodos da RTAl e RTAe, apresentados na Tabela 3, também mostraram correlação significativa entre a concentração de P no solo extraído pelas RTAl e RTAe, e a produção de massa da matéria seca das plantas de milheto $\left(r=0,857^{* *}\right)$ e $\left(r=0,782^{* *}\right)$ e para o $P$ acumulado na parte aérea e nas raízes $\left(r=0,780^{* *}\right)$ e $\left(r=0,694^{* *}\right)$, respectivamente.

Tabela 3 - Coeficientes de correlação linear de Pearson entre a quantidade de fósforo acumulado pelas plantas, os teores de $\mathrm{P}$ disponíveis no solo estimados pelos métodos Mehlich 1, Mehlich 3 e RTA, em lâminas, e RTA em esferas, e a massa da matéria seca produzida, realizado em casa de vegetação. Pearson linear correlation coeficiente between soil $P$ estimated after extraction with Mehlich 1, Mehlich 3 solution, anion exchange resin (RTA) in sheets, RTA in beads, and plant shoot dry matter.

\begin{tabular}{lccccc}
\hline & Mehlich 3 & $\begin{array}{c}\text { RTA } \\
\text { em lâminas }\end{array}$ & $\begin{array}{c}\text { RTA } \\
\text { em esferas }\end{array}$ & $\begin{array}{c}\text { Matéria } \\
\text { seca }\end{array}$ & $\begin{array}{c}\text { P } \\
\text { acumulado }\end{array}$ \\
\hline Mehlich 1 & $0,304^{\mathrm{ns}}$ & $0,290^{\mathrm{ns}}$ & $0,292^{\mathrm{ns}}$ & $0,180^{\mathrm{ns}}$ & $0,194^{\mathrm{ns}}$ \\
Mehlich 3 & & $0,789^{* *}$ & $0,942^{* *}$ & $0,914^{* *}$ & $0,806^{* *}$ \\
RTA em lâminas & & & $0,660^{* *}$ & $0,857^{* *}$ & $0,780^{* *}$ \\
RTA em esferas & & & & $0,782^{* *}$ & $0,694^{* *}$ \\
Matéria seca & & & & & $0,908^{* *}$ \\
\hline
\end{tabular}


Pela análise dos dados obtidos, verifica-se o comportamento dos métodos de extração frente às aplicações fosfatadas, no qual o método Mehlich 1 mostrou uma superestimação de $\mathrm{P}$, quando se aplicou $\mathrm{FN}$, em relação aos demais métodos avaliados, não havendo correlação com as variáveis de produção de MMS e a quantidade de $\mathrm{P}$ acumulado na planta. Esse comportamento do extrator de Mehlich 1 na superestimação do $\mathrm{P}$, quando se aplicou FN (Silva \& Raij, 1999), requer cuidados na avaliação da análise do solo (leiri et al., 2010), em que se verificou que Mehlich 1 superestimou 0 teor de $\mathrm{P}$ no solo com a aplicação de fosfatos menos solúveis. Ao contrário, o método Mehlich 3 apresentou a melhor correlação com as variáveis citadas anteriormente.

Para os métodos de Resina Trocadora de Ânions, tanto em lâminas quanto em esferas, as correlações entre os teores extraídos pelo método e a produção de MMS e P absorvido foram significativos, porém inferiores ao Mehlich 3. Como este último é de mais fácil execução em laboratório, constitui-se em um método promissor para substituir o Mehlich 1 em solos com a aplicação de fosfatos naturais.

\section{Conclusões}

O método Mehlich 1 superestima a predição da disponibilidade de fósforo para as plantas em solo com aplicação de fosfato natural de Arad, independentemente do $\mathrm{pH}$ do solo.

Os métodos Mehlich 3, Resina Trocadora de Ânions em lâminas e em esferas são mais eficientes que o Mehlich 1 na determinação de $P$ no solo, na ocasião da aplicação de Fosfato natural de Arad.

\section{Referências}

Amer F, Bouldin DR, Black CA, Duke FR (1955) Characterization of soil phosphorus by anion exchange resin adsorption and P32 equilibration. Plant and Soil 6:391-408.

Bortolon L, Gianello C, Welter S, Almeida RGO, Giasson E (2011) Simultaneous extraction of phosphorus, potassium, calcium and magnesium from soils and potassium recommendations for crops in Southern Brazil. Pedosphere 21(3):365-372.

CQFS-RS/SC (2004) Comissão de Química e Fertilidade do Solo - Manual de Adubação e de calagem para os Estados do Rio Grande do Sul e Santa Catarina. Sociedade Brasileira de Ciência do Solo. 400p.

Corrêa RM, Nascimento CWA, Freire FJ, Souza SK, Silva GB (2008) Disponibilidade e níveis críticos de fósforo em milho e solos fertilizados com fontes fosfatadas. Revista Brasileira de Ciências Agrárias 3:218-224.

Embrapa (1997) Empresa Brasileira de Pesquisa Agropecuária - Manual de métodos de análise de solo. $212 p$.
Freitas IF, Novais RF, Villani EMA, Novais SV (2013) Phosphorus extracted by ion exchange resins and mehlich-1 from oxisols (latosols) treated with different phosphorus rates and sources for varied soil-source contact periods. Revista Brasileira de Ciência do Solo $37(3): 667-677$.

Gatiboni LC, Kaminski J, Pellegrini JPR, Aquino JER (2008) Efeito da adubação fosfatada e da calagem sobre a qualidade bromatológica da forragem de pastagem natural com introdução de espécies forrageiras de inverno. Revista Brasileira de Agrociência 14(3-4):125-134.

Gatiboni LC, Kaminski J, Rheinheimer DS, Brunetto G. (2003) Superphosphate and rock phosphates as phosphorus sources for grass-clover pasture on a limed acid soil in southern Brazil. Communications in Soil Science and Plant Analysis 34:2.503-2.514.

leiri AY, Lana RMQ, Korndörfer GH, Pereira HS (2010) Fontes, doses e modos de aplicação de fósforo na recuperação de pastagem com braquiária. Ciência e Agrotecnologia 34(5):1.154-1.160.

Littel RC, Milliken GA, Stroup WW, Wolfinger RD, Schabenberger O (2006) SAS ${ }^{\circledR}$ for Mixed Models. 2.ed. SAS Institute Inc 834p.

Littel RC, Freund RJ, Spector PC (1991) SAS System for Linear Models. 3.ed. SAS Institute Inc 329p.

Mehlich A (1984) Mehlich 3 soil test extractant: A modification of Mehlich 2 extractant. Communications in Soil Science and Plant Analysis 15:1.409-1.416.

Mehlich A (1978) New extractant for soil test evaluation of phosphorus, potassium, magnesium, calcium, sodium, manganese and zinc. Communications in Soil Science and Plant Analysis 9:477-492.

Mehlich A (1953) Determination of P, Ca, Mg, K, Na and $\mathrm{NH}_{4}$ by North Carolina Soil Testing Laboratoris. Raleigh: University of North Carolina (Mimeografado).

Murphy J, Riley JP (1962) A modified single solution method for the determination of phosphate in natural waters. Analytica Chimica Acta 27:31-36.

Novais RF, Alvarez VVH, Barros NF, Fontes RLF, Cantarutti RB, Neves JCL (2007) Fertilidade do solo. Sociedade Brasileira de Ciência do Solo. 1.017p.

Novais RF, Smyth TJ (1999) Fósforo em solo e planta em condições tropicais. Universidade Federal de Viçosa 399p.

Raij BV, Quaggio JA (1983) Métodos de análise de solo para fins de fertilidade. Instituto Agronômico de Campinas. Boletim Técnico 81, 31p.

Robinson JS, Syers JK (1990) A critical evolution of the factors influencing the dissolution of gafsa phosphase rock. Journal of Soil Science 41:597-605. 
Rossi C, Anjos ARM, Camargo MS, Weber OLS, Imhoff S, Malavolta E (1999) Efeito residual de fertilizantes fosfatados para o arroz: avaliação do fósforo na planta e no solo por diferentes extratores. Scientia Agricola 56:39-46.

Santos EA, Kliemann HJ (2005) Disponibilidade de fósforo e fosfatos naturais em solos de cerrado e sua avaliação por extratores químicos. Pesquisa Agropecuária Tropical 35:139-146.

SAS (2003) Institute Inc ${ }^{\circledR}$ SAS Ver. 9.1. 3 SAS Institute Inc Lic. UDESC.

Schlindwein JA, Bortolon L, Fioreli-Pereira EC, Oliveira Bortolon, ES, Gianello C (2013) Phosphorus and potassium fertilization in no till southern Brazilian soils. Agricultural Sciences 4:39-49.

Schlindwein JA, Bortolon L, Gianello C (2011) Soil phosphorus available for crops and grasses extracted with three soil-test methods in southern Brazilian soils amended with phosphate rock. Communications in Soil Science and Plant Analysis 42(3):283- 292.
Silva FC, Raij BV (1999) Disponibilidade de fósforo em solos avaliada por diferentes extratores. Pesquisa Agropecuária Brasileira 34:267-288.

Silva FC, Raij, BV (1996) Avaliação da disponibilidade de fósforo, por diversos extratores, em amostras de solos cultivados com cana-de-açúcar. Revista Brasileira de Ciência do Solo 20(1):83-90.

Souza RM (2013) Eficiência agronômica de fosfatos de rocha, via adubação corretiva, em um cambissolo cultivado com milho no Estado de Sergipe. Universidade Federal de Sergipe (Dissertação de Mestrado em Agroecossistemas).

Steel RGD, Torrie JH, Dickey DA (1997) McGraw-Hill: Principles and procedures of statistics 3.ed. A biomerical approach 666p.

Tedesco MJ, Gianello C, Bissani CA, Bohnen $\mathrm{H}$, Volkweiss SJ (1995) Análise de solo, plantas e outros materiais. Departamento de Solos/ Universidade Federal do Rio Grande do Sul/UFRGS 174p. 